\title{
Türkiye'nin Farklı Bölgelerindeki Prangos Lindl. (Apiaceae) Cinsine Ait Taksonların Polenlerinin Morfolojik Farklılıkları
}

\author{
Birol BAŞER $^{1 *}$, Sevil PEHLIVAN ${ }^{2}$ \\ ${ }^{1}$ Bitlis Eren Üniversitesi, Fen Edebiyat Fakültesi, Biyoloji Bölümü, Bitlis, Türkiye \\ ${ }^{2}$ Gazi Üniversitesi Fen Fakültesi, Biyoloji Bölümü, Teknikokullar Ankara, Türkiye
}

\begin{abstract}
Özet
Apiaceae familyasına ait olan Prangos Lindl. 3 taksonu; İç Anadolu, Doğu ve Güney Doğu Anadolu Bölgesinde yayılış göstermektedir. Bu taksonlar 1000-2300 m arasındaki yüksekliklerde görülmektedir. Çalışmamızda Prangos cinsine ait P. pabularia Lindl. (Bitlis, Hakkâri, Elazığ, Muş, Mardin), P. platychloena Boiss. ex Tchihat. (Erzurum, Erzincan, Van), P. meliocarpoides Boiss. var. meliocarpoides (Konya, Kayseri) alınan polenlerin Wodehouse 1935 metoduna göre preparatları hazırlanmıştır. Aynı türe ait farklı bölgelerden toplanan polenlerde $\mathrm{P}, \mathrm{E}$ ve $\mathrm{P} / \mathrm{E}$ değerleri arasındaki farklılıkları saptanmıştır. Bu taksonların polenleri ışık mikroskobu ile mikrofotoğrafları çekilmiştir.
\end{abstract}

Anahtar Kelimeler: Apiaceae, prangos, polen morfoloji, Türkiye

\section{Morphological Diversity of Pollen Taxa Belongs to Prangos Lindl. (Apiaceae) Genus in Different Regions of Turkey}

\begin{abstract}
Prangos Lindl. that belongs Apiaceae family 3 taxa; Show spread in central Anatolia, East and South East Anatolia. These taxa are available at 1000-2300 m. In our study the pollen samples of $P$. pabulari Lindl. (Bitlis, Hakkâri, Elazı̆̆, Muş, Mardin), P. platychloena Boisse. ex Tchihat. (Erzurum, Erzincan, Van), $P$. meliocarpoides Boiss. var. meliocarpoides (Konya, Kayseri) were preparated according to the Wodehouse 1935 method. P, E and P/E values of the same species pollen's that collected from different regions were determined. Microphotographies of these taxa were taken with a light microscope.
\end{abstract}

Keywords: Apiaceae, prangos, polen morfology, Turkey

\section{Giriş}

Dünyada yaklaşık 300 cins ve 3000 takson içeren Umbelliferae (Apiaceae) familyası sayısı takson bakımından zengin familyalar arasındadır [1]. Yurdumuzda ise 100'den fazla cins ve 420'den fazla takson içermektedir. Apiaceae familyası Türkiye Florası'nın 4. cildinde yer almaktadır. Endemizm oranı ise \%28.1'dir [2,3]. Herrnstadt ve Heyn [4] adlı iki araştırıcının 1977 yılında yaptığı monograf çalışmasında Prangos cinsi Prangos, Intactae ve Meliocarpoides olmak üzere 3 seksiyona ayırmıştır. Çalışma konusunu oluşturan Prangos Lindl. cinsi Cachyrieae tribusunda yer alır. Prangos cinsi dünyada 24 taksonla [4]. Ülkemizde ise 11 taksonla temsil edilmektedir [2]. Son yıllarda ülkemizde yapılan çalışmalar sonucunda bu cinse $P$. heyniae ve $P$. platychloena ssp. engizekensis [5] ve $P$. turcica taksonları daha eklenmiştir [6]. Bu cinsin taksonları kalkerli kayalıklarda nadiren bazalt kayalıklarda dağ yamaçlarında ve Orta Asya'da tuzlu alanlarda yayılış göstermektedir [7]. Prangos

"Sorumlu Yazar: baser2007@gmail.com 
cinsinde göze çarpan iki önemli özellikten birincisi yeni tür teşekkülünün varlığ ikincisi çok sayıda türün çok küçük bölgede yayllış göstermesidir. $P$. ferulacea (L.) Lindl., P. pabularia Lindl., çok geniş bir yayılış alanına sahiptir [4].

Apiaceae familyasına ait Prangos cinside dahil bir çok cins üzerinde yapılan en kapsamlı polen morfolojisi çalışmaları Herrnstadt-Heyn ve Cerceau-Larrival'ın araştırmalarıdır [4, 8-13]. Bu familya ile ilgili yapılmış diğer araştımalar Erdtman [14,15], Aytuğ et al. [16], Faegri ve İversen [17], Ferreira ve Purper [18], Moore ve Webb [19], Punt [20] Bıçakcı [21], Baser [22] adlı araştırıcıların çalışmalarıdır.

Bu çalışmada, 3 farklı bölgeden (İç Anadolu, Doğu ve Güneydoğu Anadolu) ve farklı yükseklikten alınan $P$. pabularia (Bitlis, Hakkâri, Elazı̆̆, Muş, Mardin), P. meliocarpoides var meliocarpoides (Konya, Kayseri) ve P. platychloena (Erzurum, Erzincan, Van) taksonlar, P, E, L ve $\mathrm{P} / \mathrm{E}$ oranlarını aynı taksona ait farklı bölgelerden toplanan polenlerde bu değerler arasındaki farklıklar belirtilmiştir.

\section{Materyal ve Yöntem}

$\mathrm{Bu}$ çalışmada yer alan taksonlar, Gazi herbaryumu ve araziden toplanan örnekler Tablo 1'de verilmiştir. Taksonlardan alınan polenler Wodehouse [23] yöntemine göre hazırlanmıştır. Polenlerin Polar eksen (P), Ekvatoral eksen (E) ve ekvatorial çevre çapı (L) değeri ölçümleri Prior marka mikroskopta yapılmıştır. Olympus CX41 marka mikroskopta mikrofotoğrafları çekilmiştir.

Tablo 1. Prangos cinsine ait taksonların toplandığı yerler, toplayıcılar ve bulundukları herbaryum

\begin{tabular}{|c|c|c|}
\hline Taksonlar & Toplandığı Yerler & Toplayıcılar ve Herbaryum No \\
\hline P. meliocarpoides var meliocarpoides & $\begin{array}{l}\text { C5 Konya: Konya-Beyşehir yolu, } \\
\text { Altınapa Barajının doğusu 1300- } \\
\text { 1400m. }\end{array}$ & $\begin{array}{l}\text { Leg: H. Duman } \\
\text { Det: H. Duman } 8564\end{array}$ \\
\hline P. meliocarpoides var. meliocarpoides & $\begin{array}{l}\text { B5 Kayseri: Bünyan -Korumaz } \\
\text { Dağ1, istasyon tepesi, kayalık } \\
\text { yamaçlar, 1850-1900, 12/6/2000. }\end{array}$ & $\begin{array}{l}\text { Leg: M. Erkan Uzunhisarcıklı } \\
1619 \\
\text { Det: M. Erkan Uzunhisarcıklı }\end{array}$ \\
\hline P. pabularia & $\begin{array}{l}\text { B8 Muş: Hasköy- Malazgirt yolu, } \\
\text { 125.km, } 1300 \mathrm{~m} \text {, yol kenarı, } \\
6 / 6 / 2001 .\end{array}$ & $\begin{array}{l}\text { Leg: B. Başer } \\
\text { Det: B. Başer }\end{array}$ \\
\hline P. pabularia & $\begin{array}{l}\text { C8 Mardin: Mardin'den } 36 \text { km } \\
\text { sonra Gölcük Gölü yamaçları, } \\
1000 \mathrm{~m}, 6 / 6 / 2001\end{array}$ & $\begin{array}{l}\text { Leg: M. Ekici ve ark } \\
\text { Det: B.Başer }\end{array}$ \\
\hline P. pabularia & $\begin{array}{l}\text { B7 Elazı̆̆: Elazı ̆ -Bingöl 125.km, } \\
\text { yol kenar1, } 1311 \mathrm{~m}, 6 / 6 / 2001\end{array}$ & $\begin{array}{l}\text { Leg: B. Başer } \\
\text { Det: B. Başer }\end{array}$ \\
\hline P. pabularia & 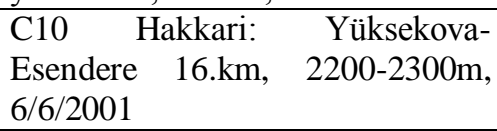 & $\begin{array}{l}\text { Leg: M. Ekici ve ark } \\
\text { Det: B. Başer }\end{array}$ \\
\hline P. pabularia & $\begin{array}{l}\text { B9 Bitlis: Bitlis-Nemrut Dağ1, } \\
\text { Şahmiran Köyü, üst kesimleri, } \\
2000 \text { m, step, 6/6/2001. }\end{array}$ & $\begin{array}{l}\text { Leg: B. Başer } \\
\text { Det: B. Başer }\end{array}$ \\
\hline P. platychloena & $\begin{array}{l}\text { B7 Erzincan: Erzincan- Kemaliye } \\
\text { Sarıkonaklar Köyü, üst kesimleri, } \\
\text { Sarıçicek Yaylası, 1500m. }\end{array}$ & $\begin{array}{l}\text { Gazi Herbaryumu } \\
\text { F. Güngör, H. Duman }\end{array}$ \\
\hline P. platychloena & $\begin{array}{l}\text { A8 Erzurum: Erzurum- İspir yolu } \\
\text { 50.km, Eğerti Köyünün kuzeyi, } \\
\text { 2000-2200m, taşlik alanlar. }\end{array}$ & $\begin{array}{l}\text { Gazi Herbaryumu } \\
\text { F. Güngör, H. Duman }\end{array}$ \\
\hline P. platychloena & $\begin{array}{l}\text { B9 Van: Van -Bahçesaray, } \\
25 . \mathrm{km} \text {, yol kenar1, } 1700 \mathrm{~m} \text {, } \\
6 / 6 / 2001 \text {, }\end{array}$ & $\begin{array}{l}\text { Leg: B. Başer } \\
\text { Det: B. Başer }\end{array}$ \\
\hline
\end{tabular}




\section{Bulgular ve Tartışma}

Prangos cinsinin taksonlarının polenlerinin ortak özellikleri şu şekildedir: Perprolat, radyal simetrili ve 3kolporat' tır. Polenlerin ekvatoryal görünüşleri subrektangulardır. Amb şekli triangular'dır. Ekzin yapıs1 tektat, ornamentasyon kutuplarda striat, ekvatorda ise rugulat'tır. Genellikle ekzin ekvatorda ve ara bölgede kalın iken kutupda incedir. Endekzin ise ara bölge ve apertür altında ektekzinden kalın iken kutupta incedir.

Bu çalışmada, Prangos cinsine ait P. pabularia Lindl. (Bitlis, Hakkâri, Elazığ, Muş, Mardin), P. platychloena Boiss. ex Tchihat. (Erzurum, Erzincan, Van), P. meliocarpoides Boiss. var. meliocarpoides (Konya, Kayseri) Aynı türe ait farklı bölgelerden toplanan bitkilerden Wodehouse 1935 yöntemine göre hazırlanan polenler 1şık mikroskobu ile ölçülerek $\mathrm{P}, \mathrm{E}$ ve $\mathrm{P} / \mathrm{E}$ değerleri arasındaki farklılıkları saptanmıştır. P. meliocarpoides var. meliocarpoides Konya ilinden toplanan örneklerden alınan, Polen trikolporat, kolpus ve por aynı düzlemde yer alır. $\mathrm{P}=33.26 \mu \mathrm{m}, \mathrm{E}=16.03$ $\mu \mathrm{m} \mathrm{P} / \mathrm{E}=2.07$, perprolat, amb çap1 $14.83 \mu \mathrm{m}$ 'dir. Polen tektat (Tablo 2) (Şekil 1 (a-b)). $P$. meliocarpoides var. meliocarpoides Kayseri ilinden toplanan örneklerden alınan, Polen trikolporat, kolpus ve por aynı düzlemde yer alır. $\mathrm{P}=34.90 \mu \mathrm{m}, \mathrm{E}=15.58 \mu \mathrm{m} \mathrm{P} / \mathrm{E}=2.24$, perprolat, amb çap 16.89 $\mu$ m'dir. Polen tektat (Tablo 2) (Şekil 1 (c-d)). P. pabularia Elazı̆g ilinden toplanan örneklerden alınan, Polen trikolporat, kolpus ve por aynı düzlemde yer alır. $\mathrm{P}=33.50 \mu \mathrm{m}, \mathrm{E}=15.79 \mu \mathrm{m} \mathrm{P} / \mathrm{E}=2.12$; perprolat, amb çap1 $15.79 \mu$ m'dir. Polen tektat (Tablo 2) (Şekil 1 (e-f)). P. pabularia Muş ilinden toplanan örneklerden alınan, Polen trikolporat, kolpus ve por aynı düzlemde yer alır. $\mathrm{P}=34.49 \mu \mathrm{m}, \mathrm{E}=$ $16.19 \mu \mathrm{m} \mathrm{P} / \mathrm{E}=2.13$, perprolat, amb çap1 $16.49 \mu \mathrm{m}$ 'dir. Polen tektat (Tablo 2) (Şekil 1 (g-h)). $P$. pabularia Mardin ilinden toplanan örneklerden alınan, Polen trikolporat, kolpus ve por aynı düzlemde yer alır. $\mathrm{P}=32.13 \mu \mathrm{m}, \mathrm{E}=14.10 \mu \mathrm{m} \mathrm{P} / \mathrm{E}=2.27$, perprolat, amb çap $15.58 \mu \mathrm{m}$ 'dir. Polen tektat (Tablo 2) (Şekil 1 (1-j)). P. pabularia Bitlis ilinden toplanan örneklerden alınan, Polen trikolporat, kolpus ve por aynı düzlemde yer alır. $\mathrm{P}=33.27 \mu \mathrm{m}, \mathrm{E}=15.36 \mu \mathrm{m} \mathrm{P} / \mathrm{E}=2.16$, perprolat, amb çap $16.79 \mu \mathrm{m}$ 'dir. Polen tektat (Tablo 2) (Şekil $1(\mathrm{k}-\mathrm{m})$ ). P. pabularia Hakkâri ilinden toplanan örneklerden alınan, Polen trikolporat, kolpus ve por aynı düzlemde yer alır. $\mathrm{P}=32.33 \mu \mathrm{m}, \mathrm{E}=15.89 \mu \mathrm{m} \mathrm{P} / \mathrm{E}=2.05$, perprolat, amb çapı $15.90 \mu$ m'dir. Polen tektat (Tablo 2) (Şekil 1 (n-o)). P. platychloena Erzincan ilinden toplanan örneklerden alınan, Polen trikolporat, kolpus ve por aynı düzlemde yer alır. $\mathrm{P}=40.87$ $\mu \mathrm{m}, \mathrm{E}=18.55 \mu \mathrm{m} \mathrm{P} / \mathrm{E}=2.20$, perprolat, amb çap1 $18.64 \mu \mathrm{m}$ 'dir. Polen tektat (Tablo 2) (Şekil 1 (p-r)). P. platychloena Erzurum ilinden toplanan örneklerden alınan, Polen trikolporat, kolpus ve por aynı düzlemde yer alır. $\mathrm{P}=43.32 \mu \mathrm{m}, \mathrm{E}=21.22 \mu \mathrm{m} \mathrm{P} / \mathrm{E}=2.04$, perprolat, amb çap1 $19.96 \mu \mathrm{m}$ 'dir. Polen tectae (Tablo 2) (Şekil 1 (s-t)). P. platychloena Van ilinden toplanan örneklerden alınan, Polen trikolporat, kolpus ve por ayn düzlemde yer alır. $\mathrm{P}=42.99 \mu \mathrm{m}, \mathrm{E}=19.93 \mu \mathrm{m} \mathrm{P} / \mathrm{E}=2.15$, perprolat, amb çapı $19.07 \mu$ m'dir. Polen tektat (Tablo 2) (Şekil 1 (v-y)).

Tablo 2. Prangos cinsine ait taksonların polenlerin morfolojik parametreleri

\begin{tabular}{|c|c|c|c|c|c|}
\hline Takson & $\begin{array}{c}\text { Yükseklik } \\
\text { (metre) }\end{array}$ & $\begin{array}{l}P(\mu \mathrm{m}) \\
\text { Ort. SD }\end{array}$ & $\begin{array}{c}\mathbf{E}(\mu \mathrm{m}) \\
\text { Ort. SD }\end{array}$ & $\mathbf{P} / \mathbf{E}$ & $\begin{array}{l}\text { L }(\mu \mathrm{m}) \\
\text { Ort. SD }\end{array}$ \\
\hline P. meliocarpoides var. meliocarpoides (Konya) & 1350 & $33.26 \pm 1.61$ & $16.03 \pm 1.34$ & 2.07 & $14.83 \pm 0.98$ \\
\hline P. meliocarpoides var. meliocarpoides (Kayseri) & $1850-1900$ & $34.90 \pm 2.42$ & $15.58 \pm 1.43$ & 2.24 & $16.89 \pm 1.00$ \\
\hline P. pabularia $($ Elazı ğ) & 1310 & $33.50 \pm 1.41$ & $15.79 \pm 1.22$ & 2.12 & $15.94 \pm 0.85$ \\
\hline P. pabularia (Muş) & $1300-1350$ & $34.49 \pm 1.35$ & $16.19 \pm 1.00$ & 2.13 & $16.49 \pm 2.01$ \\
\hline P. pabularia (Mardin) & 900 & $32.13 \pm 1.66$ & $14.10 \pm 0.76$ & 2.27 & $15.58 \pm 1.31$ \\
\hline P. pabularia (Bitlis) & 2000 & $33.27 \pm 2.35$ & $15.36 \pm 1.21$ & 2.16 & $15.79 \pm 1.05$ \\
\hline P. pabularia (Hakkari) & 2300 & $32.33 \pm 3.21$ & $15.89 \pm 0.96$ & 2.03 & $15.90 \pm 0.72$ \\
\hline P. platychloena (Erzincan) & 1500 & $40.87 \pm 2.73$ & $18.55 \pm 1.48$ & 2.20 & $18.64 \pm 1.90$ \\
\hline P. platychloena (Erzurum) & $2200-2300$ & $43.32 \pm 3.16$ & $21.22 \pm 1.33$ & 2.04 & $19.96 \pm 1.69$ \\
\hline P. platychloena $(\mathrm{Van})$ & 1700 & $42.99 \pm 2.65$ & $19.93 \pm 1.70$ & 2.15 & $19.07 \pm 2.06$ \\
\hline
\end{tabular}



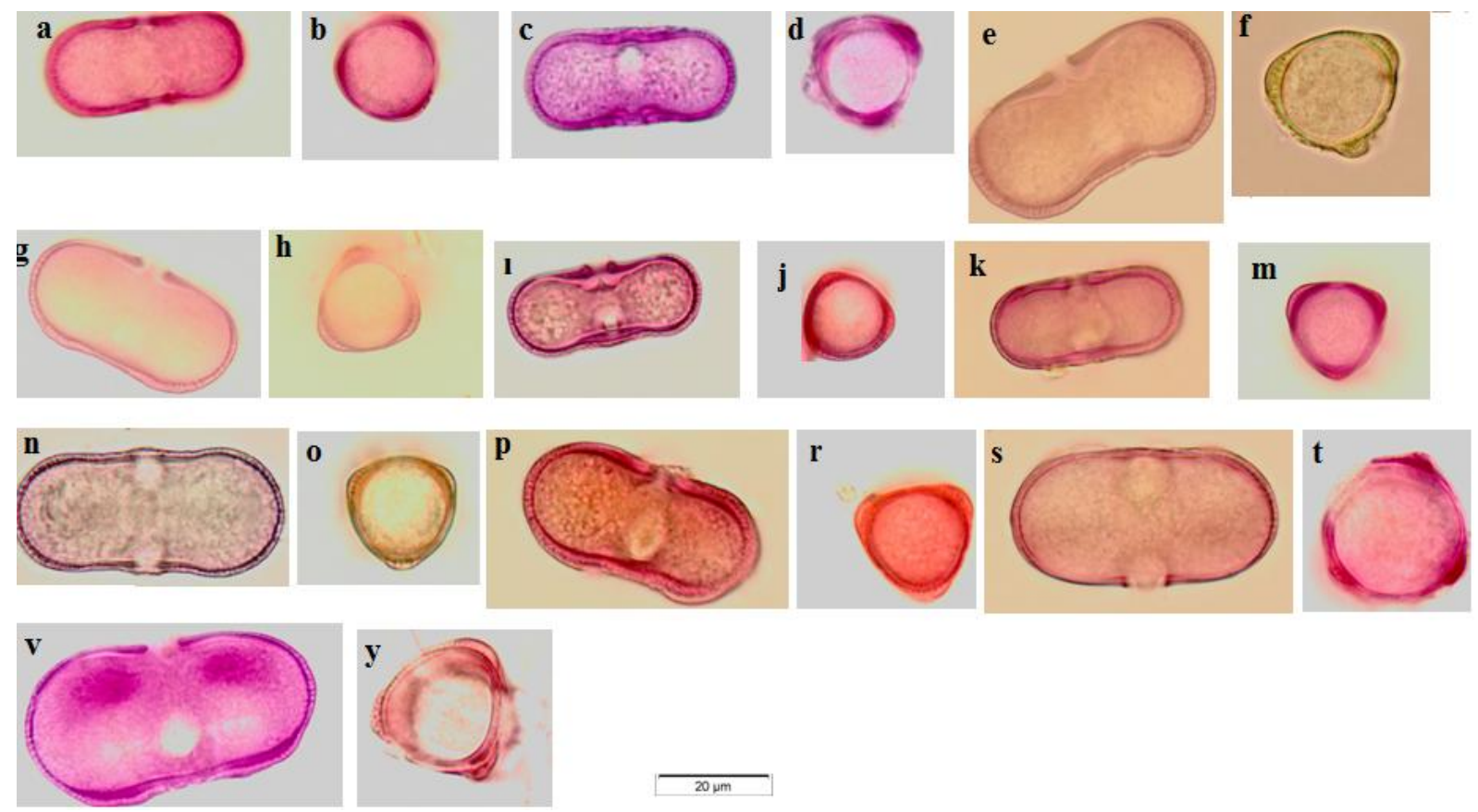

Şekil 1. Işık mikrofotoğrafları (a-b) P. meliocarpoides var. meliocarpoides (Konya), ekvatoral görünüm, polar görünüm. (c-d) P. meliocarpoides var. meliocarpoides (Kayseri), ekvatoral görünüm, polar görünüm. (e-f) P. pabularia (Elazığ), ekvatoral görünüm, polar görünüm. (g-h) P. pabularia (Muş); ekvatoral görünüm, polar görünüm. (1-j) P. pabularia (Mardin), ekvatoral görünüm, polar görünüm. (k-m) P. pabularia (Bitlis), ekvatoral görünüm, polar görünüm. (n-o) P. pabularia (Hakkâri), ekvatoral görünüm, polar görünüm. (p-r) P. platychloena (Erzincan), ekvatoral görünüm, polar görünüm. (s-t) P. platychloena (Erzurum), ekvatoral görünüm, polar görünüm. (v-y) P. platychloena (Van), ekvatoral görünüm, polar görünüm.

\section{Sonuç ve Öneriler}

Bu çalışmada, Prangos cinsine ait Prangos pabularia, P. platychloena ve $P$. meliocarpoides var. meliocarpoides taksonlarının 3 farklı bölgede (Doğu, güneydoğu ve iç anadolu) ve farklı yüksekliklede $\mathrm{P}, \mathrm{E}, \mathrm{L}$ ve $\mathrm{P} / \mathrm{E}$ oranlarının aynı takson içinde gösterdiği farklılıklar ortaya çıkarılmıştır.

Buna göre, P. meliocarpoides var. meliocarpoides taksonunun İç Anadolu Bölgesi'nde iki ilden toplanan örneklerden elde edilen P, E, L ve P/E oranları Tablo 2'de verilmiştir. Konya'da 1300 m'den toplanan örnekte P değeri $33.26 \mu$ m iken Kayseri'de 1900 m'den alınan örnekte P değeri 34.90 $\mu \mathrm{m}$ olarak tespit edilmiştir. E değeri ise Konya'daki örnekte $16.03 \mu \mathrm{m}$ iken Kayseri'deki örnekte $15.58 \mu \mathrm{m}$ bulunmuştur. P değeri yükseklik arttıkça arttığını buna karşın E değeri yükseklik arttıkça azaldığı tespit edilmiştir. L değeri ise Konya'dan alınan örnekte $16.89 \mu \mathrm{m}$ 'dir. Bu değerde de yükseklik le birlikte artış gözlenmiştir (Tablo 2). P. pabularia Doğu ve Güneydoğu Anadolu bölgesinden 5 ilden toplanan örneklerden elde edilen $\mathrm{P}, \mathrm{E}, \mathrm{L}$ ve $\mathrm{P} / \mathrm{E}$ oranları tablo-1'de verilmiştir. $\mathrm{Bu}$ taksonda Muş'ta 1300 m'de yayılış gösteren örnekte $\mathrm{P}$ değeri $34.49 \mu \mathrm{m}$ ile en yüksek değeri alırken 2300 m'de Hakkâri'de yayılış gösteren örneğin P değeri $32.33 \mu$ m'dir. Bununla birlikte 900 m'de Mardin'den alınan örnekte E değeri $14.10 \mu \mathrm{m}$ olarak en düşük değerde iken 1350 m Muş'tan alınan örnekte bu değer $16.19 \mu \mathrm{m}$ ile en yüksek değer tespit edilmiştir. $2000 \mathrm{~m}$ ve üstünde toplanan örnekte $\mathrm{E}$ değeri 15.36- $15.89 \mu \mathrm{m}$ olarak ölçülmüştür. P/E oranında ise ters orantılı olarak yükseklik arttığında bu değerin azaldığı görülmüştür (Tablo 2). L değeri ise Mardin'den 900 m'de $15.58 \mu \mathrm{m}$ ile en düşük değerde iken 2000 m'de Bitlis'teki örnekte $16.79 \mu \mathrm{m}$ ile en yüksek değere sahiptir. Bu değerde 
yükseklikle birlikte arttığı görülmüştür (Tablo 2). P. platychloena taksonunda Doğu Anadolu bölgesinde 3 ilden toplanan örneklerden elde edilen P, E, L ve P/E oranları Tablo-1'de verilmiştir. Bu taksonunda 2300 m ile en yüksek lokalitesi olan Erzurum'dan alınan örnekte P değeri $43.22 \mu \mathrm{m}$ ile en fazla değere sahip olduğunu $1500 \mathrm{~m}$ ile Erzincan'daki lokalite'de ise $40.87 \mu \mathrm{m}$ ile en düşük P değeri tespit edilmiştir. Yükseklik artı̧̧ ile bu değerin arttığı görülmektedir. E değerinde ise 1500 m'de Erzincan'daki örnekte en düşük değeri $18.55 \mu \mathrm{m}$ iken 2300 m'de Erzurum'daki lokalitede ise 21.70 $\mu \mathrm{m}$ ile en yüksek değer bulunmuştur. Yükseklikle birlikte arttığı gözlenmiştir. P/E oranı ise ters orantılı olarak yükseklik artığında bu değerin azaldığı görülmüştür. L'de ise 1500 m'de Erzincan'dan toplanan örnekte $18.35 \mu \mathrm{m}$ iken $1700 \mathrm{~m}$ Van'daki örnekte bu değer $19.96 \mu \mathrm{m}$ bulunmuştur. $\mathrm{Bu}$ değerinde yükseklikle arttığı tespit edilmiştir (Tablo 2). Yunanistan'da Centeurea L. Cinsi ile ilgili yapılmış benzer çalışmada geniş dağılımlı taksonlar ele alınmış ve farklı lokalitelerden toplanan Centeurea cinsinin 700 m'de yayılış gösteren C. affinis (Panachaikon) türünün $\mathrm{P}$ değeri $37 \pm 1.50 \mu \mathrm{m}$

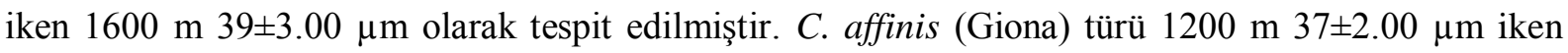

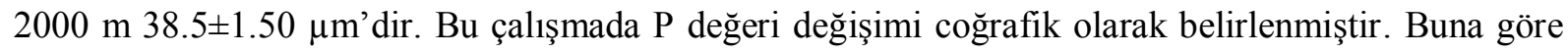
C. spinosa türünün $\mathrm{P}$ değeri Yunanistan'ın güneyinden batısına doğru arttığı belirlenmiştir. $C$. diffusa'da da bu değer kuzeyden güneye doğru arttı̆̆ gözlenmiştir. Aynı tür için farklı lokalitelerden alınan örneklerde P değerinin arttığı tespit edilmiştir [24]. Yapılan çalışmada incelenen taksonlarda P değerinde artma gözlenmiştir. Prangos cinsinin taksonlarında da batıdan doğuya doğru P, E, L değerlerinde artma olurken $\mathrm{P} / \mathrm{E}$ oranında azalma tespit edilmiştir.

\section{Kaynaklar}

1. Hickey M, King C, 1981. 100 Families of Flowering Plants. Cambridge University Press, Cambridge, London, New York.

2. Davis PH, 1972. Flora of Turkey and the East Aegean Island, Edinburgh Press, 4: 382-387.

3. Seçmen ÖY, Gemici E, Leblebici G, Görk L, Bekat, 1989. Tohumlu Bitkiler Sistematiği, Ege Univ. Fen Fak. Kitaplar serisi No: 116, İzmir.

4. Herrnsatdt I, Heyn CC, 1977. A monographic study of genus Prangos (Umbelliferae). Boissiera, 26: 911.

5. Duman H, Watson MF, 1999. Ekimia, a new genus of Umbelliferae and two new taxa of Prangos Lindl. (Umbelliferae) from southern Turkey. Edinburg Jornal Botany, 56 (2): 199-201.

6. Duran A, Sağıroğlu M, Duman H, 2005. Prangos turcica, a new species from South Anatolia, Turkey. Ann. Bot. Fennici, 42: 67-72.

7. Korovin EP, 1960. Vegetation of Central Asia. Part 1 Tashkent.

8. Cerceau MT, 1959. Cle De Determination d'Ormbellifeies De France et D'Afrique Du Nord D'Apres Leurs Grains De Pollen, Pollen et Spores, 1 (2): 145-190.

9. Cerceau MT, 1962. Le Pollen D'Ombelliferes Mediterraneennes. Pollen et Spores, 4 (1): 95-104.

10. Cerceau-Larrival MT, 1971. Morphologie Pollinique et Correlations Phylogenetiques Chez Les Ombelliferes. In: V.H. Heywood (Editör), The Biology of Chemistry of the Umbelliferae. J. Linn. Soc., Suppl., 109-156.

11. Cerceau-Larrival MT, Roland-Heydacker F, 1976. The evolutionary significance of the ultrastructure of the Exine in Umbelliferous pollen grains. In: the evolutionary Significance of the Exine. Linn. Soc. Symp., 1: 481-498.

12. Cerceau-Larrival MT, Roland-Heydacker F, Carbonnier J, 1977. Contribution palinologique a l' etude de Vanasushava pedata", Ombellifere archaique du Sud de l' Inde. Polen et Spore, 19: 285297. 
13. Cerceau-Larrival MT, Roland-Heydacker F, 1978. Apport de la palinologie a la connaissance des Ombelliferes actuellesnet fossiles. In: Actes 2e Symp. Int. Ombelliferes, Perpignan, 213-229.

14. Erdtman G, 1952. Pollen Morphology and Plant Taxanomy, Angiosperms, Almquist and Wiksell, Stockholm.

15. Erdtman G, 1969. Handbook of Palynology. Hafner Publishing Co. New York.

16. Aytuğ B, Aykut S, Merev N, Ediş G, 1971. İstanbul Çevresi Bitkilerinin Polen Atlası. İst. Univ., Yayın No. 1650, İstanbul.

17. Faegri K, Iversen J, 1975. Textbook of Pollen Analysis. Hafner Press, New York.

18. Ferreira AG, Purper C, 1972. Pollen grains of Umbelliferae from Rio Grande do Sul. III. Rev. Bras. Biol., 32: $15-19$.

19. Moore PD, Webb JA, 1983. llustrated Guide to Pollen Analysis. Hodder and Stoughton, London.

20. Punt W, 1984. The Northwest European Pollen Flora IV. Rew. of Paleobot. and Palyn, 42: 155369.

21. Başer B, 2003. Prangos Lindl. ve Ekimia H. Duman \& M.F. Watson (Umbelliferae) Cinslerine ait Bazı Taksonların Polenlerinin Morfolojik İncelemesi. Gazi Üniversitesi, Fen Bilimleri Enstitüsü, Ankara.

22. Bıçakçı A, 1996. Türkiye'de Belirlenen Echinophora L. Türlerinin Taksonomik, Palinolojisi ve Antimikrobiyal Aktiviteleri Üzerine incelemeler. Doktora Tezi, Uludağ Üniversitesi Fen Bilimleri Enstitüsü, Bursa.

23. Wodehouse R P, 1935. Pollen Grains. Mc Graw. Hill, N.Y.

24. Georgiadis T, 1985. Contribution a l'etude palynologique du genre Centraurea L. (Section acrolephus Cass.) D.C en Grece, Pollen et Spore, 37: 29-52.

Geliş Tarihi: 05/08/2015

Kabul Tarihi: 19/11/2015 